\title{
Scratch Behaviour of Silicon Solid Solution Strengthened Ferritic Compacted Graphite Iron (CGI)
}

\author{
Rohollah Ghasemi* and Anders E. W. Jarfors \\ Department of Materials and Manufacturing, School of Engineering, Jönköping University \\ P.O. Box 1026, SE-551 11 Jönköping, Sweden \\ Rohollah.Ghasemi@ju.se*, Anders.Jarfors@ju.se
}

Keywords: Silicon solution strengthening, CGI, Abrasion, Scratch testing, Scratch resistance

\begin{abstract}
The present study focuses on scratch behaviour of a conventional pearlitic and a number of solid solution strengthened ferritic Compacted Graphite Iron (CGI) alloys. This was done by employing a single-pass microscratch test using a sphero-conical diamond indenter under different constant normal load conditions. Matrix solution hardening was made by alloying with different contents of $\mathrm{Si}$; $(3.66,4.09$ and $4.59 \mathrm{wt} \%$. Si) which are named as low-Si, medium-Si and high-Si ferritic CGI alloys, respectively. A good correlation between the tensile and scratch test results was observed explaining the influence of CGI's matrix characteristics on scratch behaviour both for pearlitic and fully ferritic solution strengthened ones. Both the scratch depth and scratch width showed strong tendency to increase with increasing the normal load, however the pearlitic one showed more profound deformation compared to the solution strengthened CGI alloys. Among the investigated alloys, the maximum and minimum scratch resistance were observed for high-Si ferritic CGI and pearlitic alloys, respectively. It was confirmed by the scratched surfaces analysed using Scanning Electron Microscopy (SEM) as well. In addition, the indenter's depth of penetration value (scratch depth) was found as a suitable measure to ascertain the scratch resistance of CGI alloys.
\end{abstract}

\section{Introduction}

Pearlitic Compacted Graphite Iron (CGI) is characterised by its unique graphite morphology within the metal matrix which provides an intermediate mechanical and physical properties between the lamellar and spheroidal graphite cast irons. Hence, CGI alloys are commonly selected as suitable candidates for high mechanical and pressure applications such as diesel engine components [1]. Depending on the matrix structure, for instance pearlitic or ferritic, CGI alloys are used as piston rings and more recently as cylinder liners in heavy-fuel marine diesel engines, as well as cylinder blocks and cylinder heads in heavy-truck and heavy-fuel marine diesel engines where an excellent combination of mechanical, thermal and tribological properties are required. However, machinability of the pearlitic CGI is a big drawback [2-4]. Therefore, in recent years, CGI alloys with fully ferritic matrix have received a great attention for promising satisfactory mechanical and physical properties for the next generation of cast irons [2,5]. Thus, fully ferritic CGI alloys have been recently received more attention in industrial applications where good machinability does importantly matter, while maintaining the strength.

In piston rings and cylinder liner applications or similar situations, where the components are subjected to abrasive wear, hardness and thereby abrasion resistance is a critical factor for engine manufacturers and designers. By knowing that, the material hardness is one of the most important parameters determining abrasion resistance, improvement in hardness of the alloy may lead to a significant improvement of the abrasion resistance of the components. This can be done by applying different techniques such as pearlitic structure with finer pearlite lamellas, solid solution hardening and so onwards $[3,6]$. In the present study, Si alloy was added to strengthen the metallic matrix via a solid solution mechanism [7], increase the hardness, tensile strength, and yield strength of ferritic matrix $[8,9]$. However, this aim will be reached in expense of, to some extent, losing thermal conductivity and ductility. To have a better understanding and precise macroscopic scale 
explanation of the elastic and plastic deformation of a heterogeneous material such as CGI, it is necessary to do first a proper local investigation using micro-mechanical models [10]. To do so, several previous in-situ SEM studies were carried out by Murray et al. [11], Glaeser [12], Bates et al. [13], Kayaba et al. [14, 15], and Calabrese et al. [16] to elucidate the macroscopic abrasive wear by scrutinising in microscopic scale on different materials such as steel and polymers. However, a very few studies have been performed on cast iron materials in general and CGI in particular.

Over the last two decades, scratch behaviour of materials has gradually become an important research topic in both the academic and industrial world to assess the abrasion resistance of materials $[17,18]$. However, it is crucial to identify the key parameters compared to the reality and mimic the real abrasion condition, instrumented scratch testing has been shown to be a useful tool for characterisation of the abrasion resistance of materials [19]. This study presents an experimental simulation of a rigid sphero-conical indenter sliding against a pearlitic and a number of fully ferritic CGI alloys which have been solution strengthened by different Si contents. To determine the abrasion behaviour and characterise the relative scratch resistance compared to the normal pearlitic CGI, single-pass microscratch test was employed as presented by Kato and Hokkirihgawa [20]. The influence of scratch normal load on tribological behaviour has been discussed. The results obtained from microscopic analysis are used to make correlation between the abrasive wear modes, coefficient of friction and scratch deformation mechanisms of CGI alloys with various microstructures.

\section{Experimental Procedure}

\subsection{Materials and Microstructural Analysis}

Four grades of CGIs were investigated in this study. Three ferritic CGI solution strengthened with different Si levels 3.66, 4.09 and $4.59 \mathrm{wt} \%$, named as low-Si, medium-Si and high-Si, respectively and one pearlitic CGI used as "reference sample". A typical composition of the materials used in this study is given in Table 1.

Table 1. Chemical composition of CGI samples investigated (wt.\%).

\begin{tabular}{|c|c|c|c|c|c|c|c|c|c|c|c|c|}
\hline Materials & $\mathrm{C}$ & $\mathbf{S i}$ & Mn & $\mathbf{P}$ & $\mathbf{S}$ & $\mathrm{Cr}$ & Mo & $\mathbf{N i}$ & Co & $\mathbf{C u}$ & V & Mg \\
\hline $\begin{array}{l}\text { Pearlitic } \\
\text { CGI }\end{array}$ & 2.73 & 04 & 0.420 & 0015 & 00 & 033 & 007 & 0.038 & 0.019 & 0.791 & 0.011 & 0.020 \\
\hline Low-Si & 3.24 & 3.66 & 0.202 & 0.010 & 0.011 & 0.054 & 0.010 & 0.065 & 0.025 & 0.020 & 0.016 & 0.014 \\
\hline $\begin{array}{l}\text { Medium- } \\
\text { Si }\end{array}$ & 3.01 & 4.09 & 0.190 & 0. & 0. & 0.041 & 10 & 6 & 0.025 & 0.019 & 0.016 & 0.010 \\
\hline High-Si & 3.13 & 4.59 & 0.173 & 0.010 & 0.009 & 0.038 & 0.010 & 0.066 & 0.027 & 0.019 & 0.015 & 0.014 \\
\hline
\end{tabular}

The scratch specimens were first metallographically ground according to the standard sample preparation procedure for cast iron materials followed by polishing with a diamond suspension down from 3 to $1 \mu \mathrm{m}$. The samples were finally cleaned with acetone in an ultrasonic cleaner prior to scratch testing. The surface prepared had a surface roughness ( $\mathrm{Ra}$ ) of approximately $0.02 \mu \mathrm{m}$. In addition, in case where the deformation of the pearlitic structure was of interest, the polished surface was very gently etched using Nital solution $2 \%$ before scratching. Scanning Electron Microscopy (SEM) was utilised to investigate both morphology and scratch deformation mechanisms.

\subsection{Tensile Testing and Hardness Measurements}

Tensile test was made to determine mechanical properties, including Young's modulus E, offset proof stress $R_{p 0.2}$, tensile strength $R_{m}$, and elongation to failure $\left(\varepsilon_{\mathrm{F}}\right)$ of the specimens. The tests were carried out according to the tensile testing standard ISO 6892-1:2009 method B at ambient temperature.

For all the investigated materials, Vickers microhardness measurements were carried out locally under applied load of $6 \mathrm{~N}$ just over the pearlite colonies and ferritic matrix following the ISO 6507- 
1:2005 standard. In addition, Brinell measurements were made according to standard ISO 65061:2005 using a load of $750 \mathrm{~kg}$ on a $5 \mathrm{~mm}$ taken tungsten carbide ball. An average of at least 10 measurements is reported for each hardness value in this study.

\subsection{Microscratch Testing}

In the present investigation, a sphero-conical diamond indenter with a conical tip (radius $50 \mu \mathrm{m}$ ) and induced cone angle of $90^{\circ}$ was used for scratching the surfaces over $1000 \mu \mathrm{m}$ with scratch velocity of $10 \mu \mathrm{m} / \mathrm{s}$. The microscratch tests were conducted under constant normal load conditions at room temperature $\sim 25^{\circ}$. In addition to the microscratch by applying the main load, the pre- and post-scan scratches were made at very low load condition of about $0.2 \mathrm{mN}$ in the same direction of the main scratches. The microscratch depth was recorded continuously during scratching. The microscratch width was then calculated based on the data obtained from the indenter's depth of penetration during scratching. Considering the sphero-conical indenter tip geometry, Eq. 1 was used to calculate the microscratch width $W$ during scratching [21]:

$$
W=\left(2 R h-h^{2}\right)^{1 / 2}
$$

where $W, h$ and $R$ are the scratch width, scratch depth and radius of the tip of indenter, respectively.

\section{Results and Discussion}

\subsection{Microstructural Characterisation}

Fig. 1(a) represents the typical LOM image of the pearlitic CGI sample which contains mainly pearlite, while the new produced solution strengthened CGI consisted of compacted graphite particles and some graphite nodules within a fully ferritic matrix, as shown in Fig. 2(b).
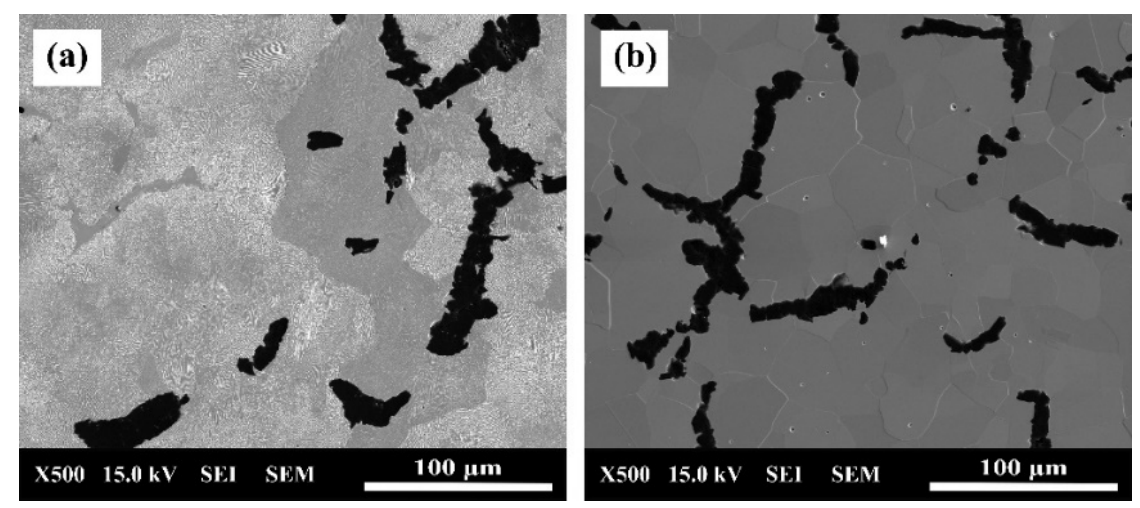

Figure 1. SEM micrographs representing the typical CGI materials used in this study; (a) pearlitic, (b) fully ferritic solution strengthened (etchant Nital 2\% solution).

\subsection{Tensile Properties and Hardness Measurements}

The influence of Si addition on hardness and tensile properties of the CGI examined samples is summarised in Table 2. The microhardness measurements were performed only over the pearlite colonies for the pearlitic CGI and the ferritic matrix for the solution strengthened ferritic CGI alloys. As is shown in Table 2, no significant difference was observed in Young's modulus of low-, medium, and high-Si alloys. Increasing of Si content increased both tensile and yield strength, however, it was more significant for Rp0.2 than that of $\mathrm{Rm}$ with increase of approximately $29 \%$, and 25\% with increasing Si content from low- to high-Si level. In contrast, silicon addition, resulted in decrease of elongation to failure of the ferritic solution strengthened CGI materials at room temperature $\sim 25^{\circ} \mathrm{C}$. 
Table 2. Hardness and tensile properties of the investigated CGI materials.

\begin{tabular}{lcccccc}
\hline \multirow{2}{*}{ Materials } & \multicolumn{9}{c}{ Mechanical properties } \\
\cline { 2 - 7 } & $\begin{array}{c}\text { Microhardness } \\
{[\mathbf{H V}]}\end{array}$ & $\begin{array}{c}\text { Brinell Hardness } \\
{[\mathbf{H B W} \text { 5/750] }}\end{array}$ & $\begin{array}{c}\mathbf{E} \\
{[\mathbf{G P a}]}\end{array}$ & $\begin{array}{c}\mathbf{R}_{\mathbf{p 0 . 2}} \\
{[\mathbf{M P a}]}\end{array}$ & $\begin{array}{c}\mathbf{R}_{\mathbf{m}} \\
{[\mathbf{M P a}]}\end{array}$ & $\begin{array}{c}\boldsymbol{\varepsilon}_{\mathbf{F}} \\
{[\mathbf{\%}]}\end{array}$ \\
\hline Pearlitic CGI & 219 & 207 & 145 & 312 & 410 & 1.28 \\
Low-Si & 230 & 182 & 153 & 367 & 409 & 2.18 \\
Medium-Si & 258 & 198 & 151 & 413 & 447 & 1.53 \\
High-Si & 287 & 223 & 151 & 468 & 503 & 1.06 \\
\hline
\end{tabular}

The increased strength of the Si-alloyed material was related to the solution strengthening effect of $\mathrm{Si}$ which increased the hardness of the matrix. This effect can be seen in both Vickers microhardness and Brinell hardness measurements listed in Table 2. As it is observed, the different contents of $\mathrm{Si}$ in the matrix provided various ranges of microhardness. The hardness measurements displayed a slight increase in matrix Vickers microhardness values as well, from approximately 230 $\mathrm{HV}$ to $287 \mathrm{HV}$, along with an increase in Si content from low- up to high-Si level, which corresponds to an increase of roughly $25 \%$ in microhardness value, as given in Table 2 . In a similar manner, the Brinell hardness of the solution strengthened CGI alloys was increased by about $18 \%$ with increase of Si content from low- to high- content. This suggests that most of the increase obtained in strength could be attributed to the Si solid solution hardening mechanism.

\subsection{Microscratch Constant Load Testing}

Figs. 2(a) and (b) indicate the deformation of low-Si solution strengthened and conventional pearlitic CGI alloys under a constant normal load of $600 \mathrm{mN}$ after scratching. As can be seen, under such a loading condition, the scratch depth would be about $1 \mu \mathrm{m}$ which almost no considerable pileup formation.

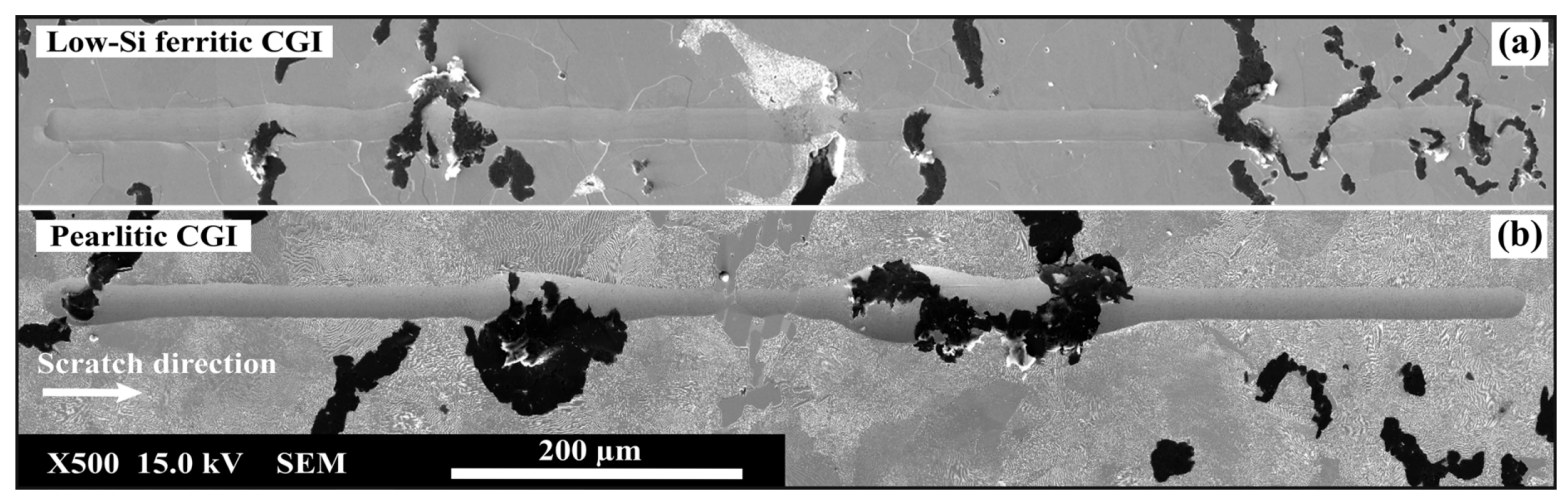

Figure 2. Scratched surfaces under $600 \mathrm{mN}$ constant load condition correspond to (a) low-Si ferritic solution strengthened, (b) pearlitic CGI investigated alloys.

For all materials, the scratch depth and scratch width tend to increase with increasing the applied normal load as shown in Figs. 3(a) and (b). The scratch depth and scratch width values presented in Fig. 4 correspond to the six measurements positions along the scratch length. Three scratches are made for each individual applied normal load. The mean value of all positions is summarised for the investigated materials in Figs. 3(a) and (b). Note should be taken that the microscratch depth data presented in Fig. 3(a) are corresponding to the recorded data during the scratching. The greatest scratch depth was found for pearlitic CGI alloy followed by low-, medium- and high-Si alloys. The latter both show a significantly shallower scratch depth. Below the $600 \mathrm{mN}$, pearlitic and low-Si CGI's showed very close behaviour, however increasing the scratch load to $1000 \mathrm{mN}$ resulted in more profound matrix deformation and scratch depth in pearlitic CGI studied sample compared to the ferritic solution strengthened CGI alloys. 


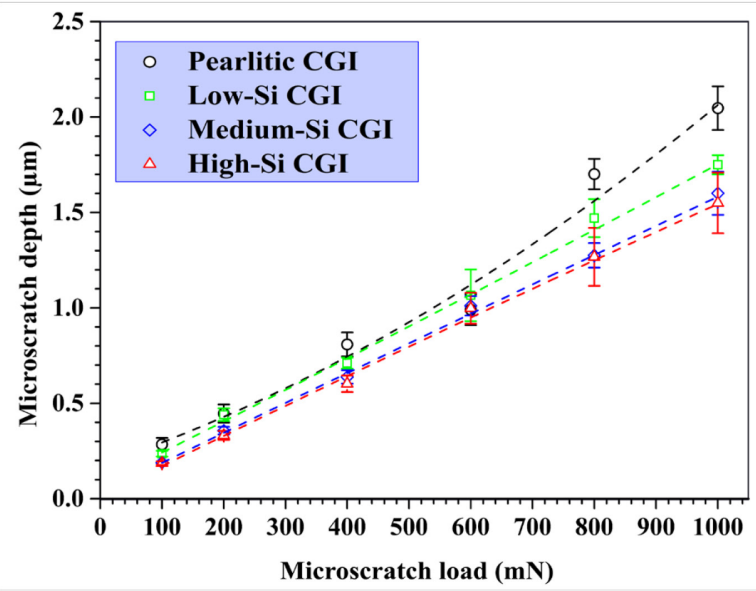

(a)

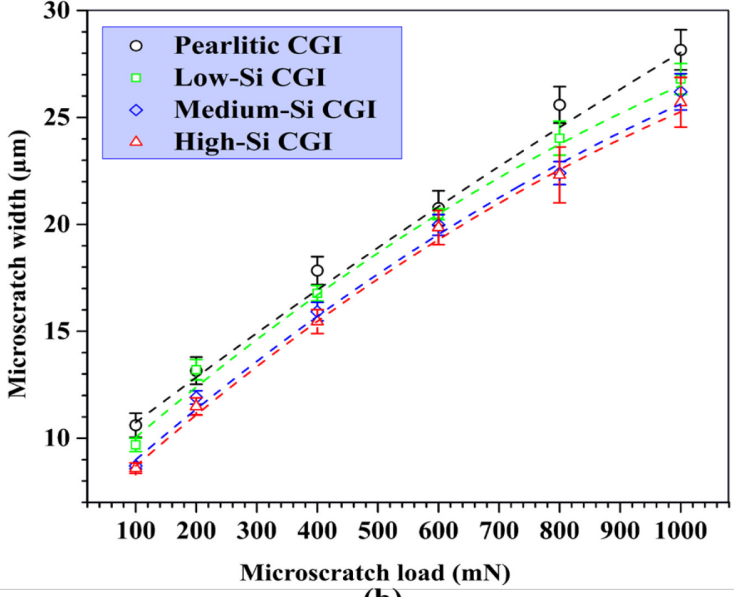

(b)

Figure 3. (a) Microscratch depth, and (b) microscratch width corresponding to the constant load scratch testing ranging from $100 \mathrm{mN}$ to $1000 \mathrm{mN}$.

Furthermore, as it is obvious from Fig. 3(a), the microscratch depth profiles for medium- and high-Si content showed very close values for each range of applied load condition and the difference is almost insignificant. The microscratch depth of high-Si alloy is only slightly lower than that of medium-Si one. A lower scratch depth suggests a greater resistance to scratching with a reduction in material loss. This behaviour shows a good correlation between tensile and scratch test results, presented in Table 2 and Fig. 3, explaining the influence of matrix hardness on scratch behaviour of CGI, in that high-Si CGI alloys having the largest microhardness and Brinell hardness showed higher scratch resistance than the medium-, low-Si fully ferritic solution strengthened and pearlitic CGI's.

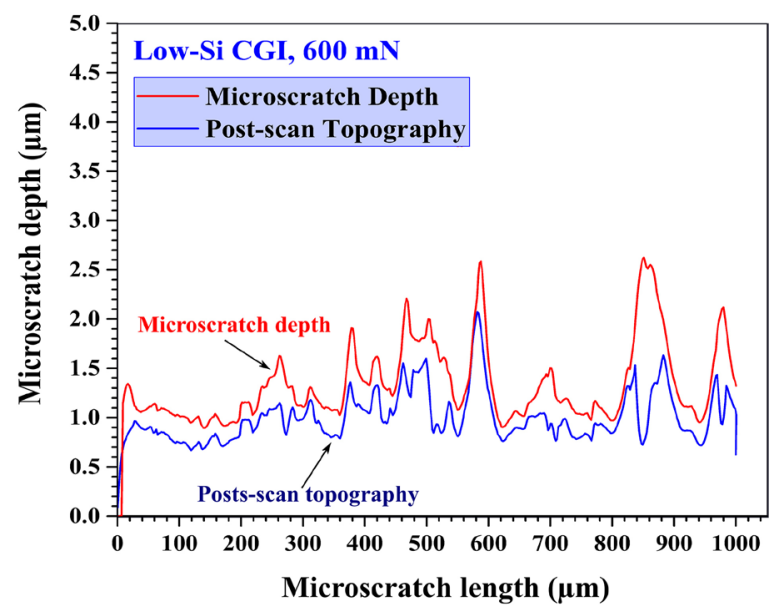

(a)

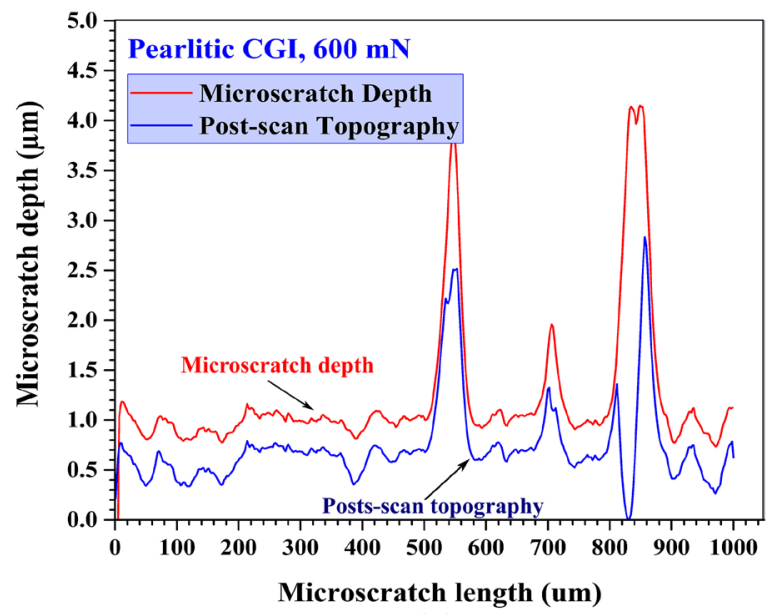

(b)

Figure 4. Microscratch depth profiles correspond (a) Low-Si ferritic solution strengthened, (b) a typical pearlitic CGI under $600 \mathrm{mN}$ applied constant load conditions.

Figs. 4(a) and (b) show the microscratch depth measured during scratching under scratch load of $600 \mathrm{mN}$ for low-Si and pearlitic CGI's, respectively. The difference between post-scan topography and microscratch depth profiles can be used as an indication of the significance of the elastic recovery taking place after removing the indenter in each material with its characteristic structure. As can be observed, a larger elastic recovery is involved in pearlitic CGI compared to low-Si ferritic alloy under $600 \mathrm{mN}$ applied load. Similar trend was observed for medium- and high-Si alloys as well. So that, increasing Si content resulted in less gap between the post-scan topography and microscratch depth curves. Thus, this is translated to the less elastic recovery happening after scratching. Such behaviour can be related to a combination effect of Young's modulus, hardness and nature of the materials, in that, pearlitic CGI with lowest E showed the largest elastic recovery and high-Si the lowest among the investigated samples. 
Fig. 5 shows SEM micrographs of typical microscratches made over pearlitic and solution strengthened ferritic CGI specimens under various load conditions. For pearlitic CGI, applying load of $100 \mathrm{mN}$, produced a scratch with small displacement of the matrix, while a less deformation was observed for low-Si CGI. However, this load was insufficient to produce significant scratch on high-Si alloys, and that was confirmed by the absence of visible grooves produced over the surface.
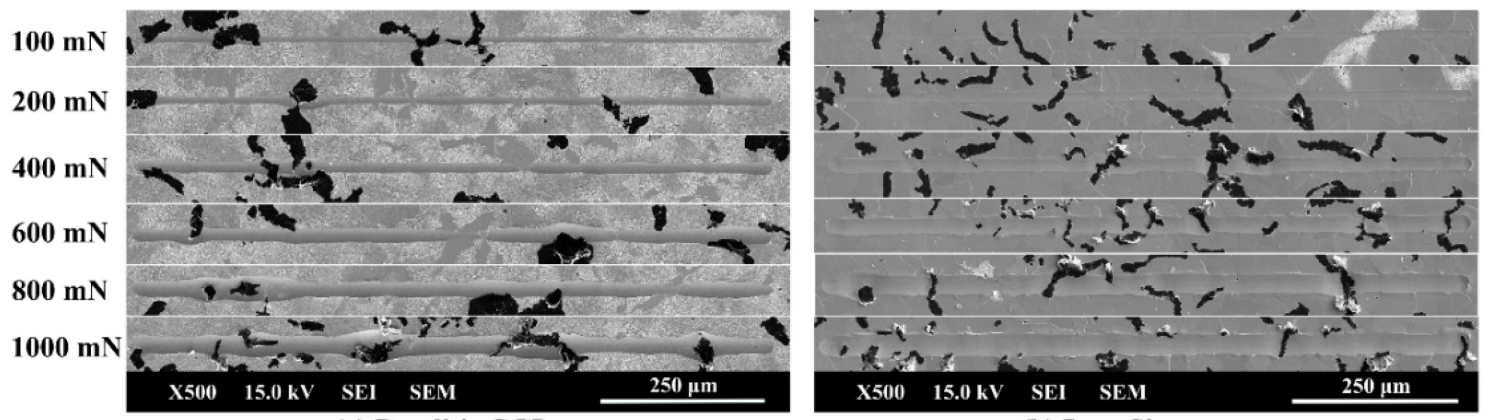

(a) Pearlitic CGI

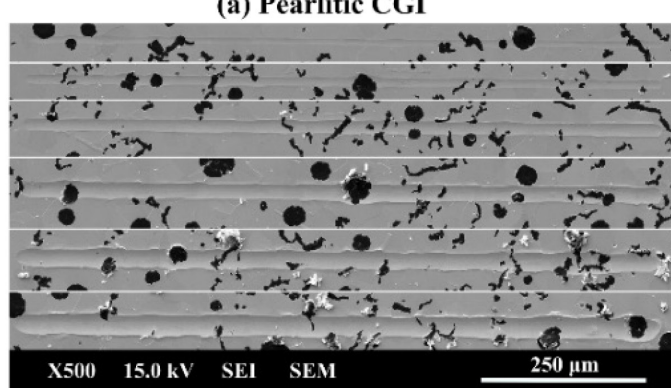

(c) Medium-Si

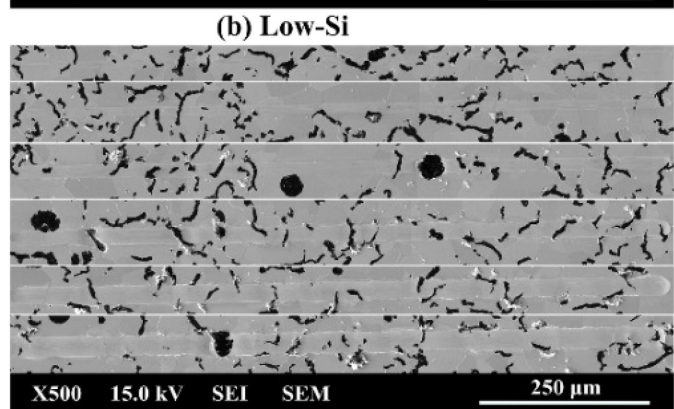

(d) High-Si

Figure 5. SEM micrographs of scratched CGI materials investigated under various normal load magnitudes:

(a) pearlitic, (b) low-Si, (c) medium-Si, (d) high-Si alloys.

In addition, for all the materials, it was observed that the deformation mechanism of the matrix is primarily controlled by a gentle increase of penetration depth of the indenter under low load conditions i.e. 100, 200 and $400 \mathrm{mN}$. However, for scratches, performed at normal loads higher than that of $600 \mathrm{mN}$, a more profound plastic deformation was observed showing a transition in wear mode from very smooth micro-ploughing to moderate micro-ploughing. This happening was primarily accompanied by displacement of metal matrix; however, no significant pile-up formation could be detected. Further increase of normal load up to $1000 \mathrm{mN}$ resulted in a considerable change of micro-mechanism from micro-ploughing to micro-wedge formation where the matrix deformation is expressed by the appearance of pile-up on the edge of microscratch grooves and small chip formation in front of the indenter. A clear decrease exists for transition from microploughing to micro- wedge forming mode with increasing of the matrix hardness which is in accordance with literature [22].

Table 3. Coefficient of friction measured during scratching of pearlitic, low-, medium-and high-Si CGI alloys under applied constant normal loads ranging from $100 \mathrm{mN}$ up to $1000 \mathrm{mN}$.

\begin{tabular}{lcrcr}
\hline Scratch load $(\mathbf{m N})$ & Pearlitic CGI & Low-Si & Medium-Si & High-Si \\
\hline $\mathbf{1 0 0}$ & $0.19 \pm 0.010$ & $0.12 \pm 0.011$ & $0.12 \pm 0.001$ & $0.12 \pm 0.003$ \\
$\mathbf{2 0 0}$ & $0.20 \pm 0.006$ & $0.16 \pm 0.004$ & $0.14 \pm 0.001$ & $0.14 \pm 0.003$ \\
$\mathbf{4 0 0}$ & $0.21 \pm 0.003$ & $0.18 \pm 0.003$ & $0.17 \pm 0.002$ & $0.16 \pm 0.002$ \\
$\mathbf{6 0 0}$ & $0.22 \pm 0.017$ & $0.20 \pm 0.003$ & $0.19 \pm 0.006$ & $0.18 \pm 0.002$ \\
$\mathbf{8 0 0}$ & $0.23 \pm 0.005$ & $0.21 \pm 0.022$ & $0.20 \pm 0.003$ & $0.19 \pm 0.001$ \\
$\mathbf{1 0 0 0}$ & $0.23 \pm 0.016$ & $0.22 \pm 0.009$ & $0.21 \pm 0.001$ & $0.20 \pm 0.007$ \\
\hline
\end{tabular}

Moreover, it was found that the difference in lubrication mechanism explains the higher friction and also the differences in machinability between compacted and nodular graphite irons [2, 3]. This can be partially explained by the role of the graphite in lubricating the surface during scratching 
(micro-cutting). As can be seen, for instance, scratching of medium-Si alloy under $800 \mathrm{mN}$ constant load, the compacted graphite particles are fractured and extruded, while the nodular graphite particles stayed almost intact although the scratches were made very close by.

Moreover, it was also found that increasing scratch load resulted in severe deformation modes in all investigated materials, however the largest and lowest influence and matrix failures were seen for pearlitic and high-Si CGI alloys, respectively. Similar behaviour was observed in pile-up tendency. Coupling the microscratch depth, width and hardness, it can be concluded that due to the lower scratch depth and smaller pile-ups, scratching of high-Si results in lower tangential forces and friction coefficients. This occurrence is in accordance with the coefficient of friction data presented in Table 3, where the lowest friction coefficient was achieved for high-Si CGI alloys, although the scratch depth is only slightly lower compared to low- and medium-Si alloys. Note should be taken that, SEM images of microscratch showed more uniform grooves rather than the pearlitic CGI, this is mainly related to the uniform hardness of the solution strengthened ferritic matrix. This may result in considerable improvement in machinability of these alloys which was discussed in our previous study [를.

\section{Conclusions}

As-cast compacted graphite iron samples with various microstructures were investigated for differences in matrix hardness, mechanical properties and scratch behaviour. It was observed that the scratch behaviour of the studied CGI alloys followed, in principle, the same trend as it has been observed in hardness measurements. So that a clear decrease was observed for transition from micro-ploughing to micro-wedge formation mode accompanied with increasing of matrix hardness. In addition, the scratch depth and width were found as suitable measures to ascertain the scratch resistance of the investigated CGI alloys.

\section{Acknowledgements}

The authors kindly acknowledge the financial support of KK-Foundation under the ProSpekt2016 scheme no. 20170021, Vinnova under FFI-program no. 2012_137 2.4.2, and CompCAST no. 20100218. Swerea SWECAST, Volvo Powertrain, Nya Arvika Gjuteri AB and SKF Mekan AB are greatly acknowledged for their support.

\section{References}

[1] S. Dawson, F. Indra, Compacted graphite iron-A new material for highly stressed cylinder blocks and cylinder heads, Fortschritt-Berichte VDI, 639 (2007) 181.

[2] S. Dawson, I. Hollinger, M. Robbins, J. Daeth, U. Reuter, H. Schulz, The Effect of Metallurgical Variables On the Machinability of Compacted Graphite Iron, SAE Transaction, 110 (2001) 334-352.

[3] A. Berglund, C.M. Nicolescu, H. Svensson, The effect of interlamellar distance in pearlite on CGI machining, in: International Conference on Mechanical Engineering, 2009, pp. 33-44.

[4] G. Grenmyr, A. Berglund, J. Kaminski, C.M. Nicolescu, Investigation of tool wear mechanisms in CGI machining, IJIDEM, 4 (2011) 3-18.

[5] H.C. Shin, High-Silicon Ferritic Compacted Graphite Cast Iron Having High-Temperature Strength and High Oxidation-Resistance, in, Google Patents, 2007.

[6] M. König, M. Wessén, Influence of Alloying Elements On Microstructure and Mechanical Properties of CGI, Int. J. Cast Metal Res., 23 (2010) 97-110.

[7] Z. Fan, Y. Wang, S. Liang, H. Huang, Strengthening Cast Iron Matrix by Alloying Elements with Positive and Negative Segregation, Acta Metall Sin-Engl., 10 (1997) 329-332.

[8] H.S. Desai Gowda, P.G. Mukunda, M. Herbert, Correlation of Tribological Properties with Microstructure and Mechanical Properties of Graphite Cast Irons Centrifugally Cast for Engine Liner, Trans Indian Inst Met, (2014) 1-10. 
[9] D.M. Stefanescu, J.R. Davis, J. Destefani, Metals Handbook, Vol. 1, Properties and Selection: Irons, Steels and High Performance Alloys, in: ASM International, Materials Park, Ohio, USA, 1990, pp. 100-120.

[10] L. Collini, Micromechanical modeling of the elasto-plastic behavior of heterogeneous nodular cast iron, doctarat en genie industrilel, Universita'Degli Studi di Parma, (2004).

[11] M. Murray, P. Mutton, J. Watson, Abrasive wear mechanisms in steels, J Tribol, 104 (1982) 916.

[12] W. Glaeser, Wear experiments in the scanning electron microscope, Wear, 73 (1981) 371-386.

[13] T. Bates Jr, K.C. Ludema, W. Brainard, A rheological mechanism of penetrative wear, Wear, 30 (1974) 365-375.

[14] T. Kayaba, K. Kato, Y. Nagasawa, Abrasive wear in stick-slip motion, in: Proc. Int. Conf. on Wear of Materials, San Francisco, CA, 1981, pp. 439.

[15] T. Kayaba, K. Hokkirigawa, K. Kato, Analysis of The Abrasive Wear Mechanism by Successive Observations of Wear Processes in A Scanning Electron Microscope, Wear, 110 (1986) 419-430.

[16] S.J. Calabrese, F.F. Ling, S.F. Murray, Dynamic wear tests in the SEM, ASLE Trans., 26 (1983) 455-465.

[17] L.A. Franco, A. Sinatora, Material removal factor (fab): A critical assessment of its role in theoretical and practical approaches to abrasive wear of ductile materials, Wear, 382 (2017) 51-61.

[18] J.L. do Vale, M. Cortz, V.M.S. Bertolini, C.H. da Silva, G. Pintaude, Comparison of scratch resistance of lamellar and compacted graphite irons used in cylinder liners, J Braz Soc Mech Sci \& Eng, (2016).

[19] V. Jardret, H. Zahouani, J.L. Loubet, T.G. Mathia, Understanding and quantification of elastic and plastic deformation during a scratch test, Wear, 218 (1998) 8-14.

[20] K. Kato, K. Hokkirigawa, Abrasive Wear Diagram, Proc. Eurotrib, 85 (1985) 1-5.

[21] S.K. Sinha, D.B. Lim, Effects of normal load on single-pass scratching of polymer surfaces, Wear, 260 (2006) 751-765.

[22] K. Hokkirigawa, K. Kato, Z. Li, The Effect of Hardness On the Transition of the Abrasive Wear Mechanism of Steels, Wear, 123 (1988) 241-251.

[23] R. Ghasemi, The influence of microstructure on mechanical and tribological properties of lamellar and compacted irons in engine applications, in: Department of Materials and Manufacturing, School of Engineering, Jönköping University, Sweden, 2016, pp. 188. 